is ever so in a new country to the first explorers. I | only hope to point to the way, go as far as I can, and trust others better equipped, and time, will open up the way and reap the harvest. So, in concluding these suggestive thoughts, I recapitulate the pertinent point, that normal nutrition is to physiology what abnormal nutrition is to pathology.

The chairman then announced the following Executive Committee for the Section: Dr. II. A. Hare, Philadelphia; Dr. I. E. Atkinson, Baltimore; Dr. N. S. Davis, Jr., Chicago.

Third Day-June 9-Mornixg Sessiox.

A paper was read entitled

\section{THE CARDIAC INDICATIONS AND CONTRA- INDICATIONS IN THE TREATMENT OF PNEUMONIA.}

BY J. M. ANDERS, M.D., PhD., PROFESSOR OF THEORY AND PRACTICE OF MEDICINE AND CLINICAI MED-
ICINE, MEDICO-CHIRURGICA, COLIEGE, PHILA.

It is a well known fact that in acute lobar pneumonia failure of heart power is the immediate cause of death, as a rule.

In the first place there are three leading factors which enter into the causation of cardiac-insufficiency in this disease, which factors may operate singly or in combination. They may be conveniently considered under as many different heads, to-wit:

First. The heart failure may be due to the toxic effects of the ptomaines present in the blood, on the heart muscle and ganglia.

Secondly. It may be due in part at least to the obstruction offered to the pulmonary circulation by the inflammatory exudate in the air vessicles.

Thirdly. The formation of cardiac thrombi may lead to right ventricular dilatation and exhaustion of heart power.

Preumonia belongs to the acute infectious diseases, hence it is intelligible that on account of the overwhelming of the system by the ptomaines produced by the pneumococcus, the heart may become exhausted. It is not an uncommon event in the experience of the busy practitioner to meet, witly instances of this malignant and fatal type of the affection. These cases can as a rule, be recognized during the progress of their brief course. Auscultation of the heart reveals a feeble, short, first sound, associated usually with a feeble aortic and pulmonic second sound. The pulmonary artery second sound is sometimes accentuated when these cases reach the stage of complete consolidation, though most generally for a brief period only. But though the heart power fails under these circumstances there are few special indications presented by the organ for treatment.

Alcohol in moderate doses is of the first importance since it aids the digestion, imparts vigor to the heart muscle, stimulates respiration and the nerve centers. We should supplement the action of the alcohol by the administration of strychnia which stimulates, more particularly, the cardiac nerve ganglia, the vasomotor and the respiratory centers. Digitalis is to be employed cautiously, indeed, is only useful when the pulse becomes very frequent as well as irregular or intermittent. Under these circumstances digitalis, by lengthening the period of diastole, facilitates the nutrition of the cardiac muscle.

In this connection the investigations of Drs. G. and F. Klemperer, carried out to ascertain among other things, whether poeumonia can be cured by the blood serum of immune animals, or those that have recovered from the disease, are of considerable interest and importance.

Their experiments "confined to rabbits show that the inoculation of any nutrient medium, in which the pneumococcus has been cultivated will protect an animal against pneumonic septicæmia. Again, serum from animals enjoying immunity cured pneumonic septicæmia, especially when introduced into the circulation, and this in 24 hours, as a rule. It was found that when the pneumococcus enters the body of an animal it generates a poison 'pneumotoxine' which can be isolated: this sets up a febrile condition, lasting several days, by which time another substance 'anti-pneumotoxine' is formed, and through its influence cures pneumonic septicæmia."

The drugs employed, in the most prevalent methods of combating the local engorgement of the lung in the first stage of pneumonia, for their influence upon high temperature to some extent, as well as upon the heart, are veratrum viride, aconite, and antimony. To the depressing effect on the heart of the pneumonic poison, the majority of physicians are still in the habit of adding the depressing influence of cardiac sedatives. Now whilst I am willing and ready to concede to the facts, that veratrum viride and aconite act as vaso-motor depressants, quiet the heart and relax the blood-vessel walls, I affirm without hesitation that they do so at the expense of cardiac power,-a thing to be scrupulously avoided. Again, it is undeniably true that if the exhibition of arterial depressants do not abort the disease promptly, then an additional load has been imposed upon the heart, whilst the pathological changes, owing to the fact that this disease rapidly undermines the vital forces, have received new impetus.

To attempt to relieve engorgement of the lung is justifiable, and this can frequently be most safely accomplished by local means, especially cupping and leeching or by the application of the jce box. The excited action of the heart which tends to keep up the pulmonary engorgement may be controlled by the use of morphine which also allays nervous excitement and pain without depressing the central organ of circulation. In sthenic types of the affection moderate bleedings from the median basilic vein would meet the same indications more promptly, and with less danger from ulterior deleterious effect upon the heart than by the use of such remedies as aconite, veratrum viride, antimony, etc.

Prof. H. C. Wood, ${ }^{1}$ contends that the admin. istration of veratrum viride bleeds the patient into his own abdomen, leaving the blood to be utilized subsequently when needed; and in this fact, he tells us, lies the advantage of veratrum over venesection. This noted observer admits that this agent depresses the heart, but claims that it does not exhaust it. Now it seems to me that to depress the heart which is already over-burdened, even for a couple of days, must tend to "exhaust it." Occasionally cases are met with in which there is a comparatively large area of lung tissue consolidated with a disproportionately mild type of systemic infection. The heart acts regularly, the first sound is good and the pulmonary second sound is accentuated. In these instances arterial sedations ar $\theta$ not so fruitful of evil results, but they recover all the more promptly when the use of this class of remedial agents is omitted. 
In the severer forms of acute lobar pneumonia, the temperature it is well known, is often high and rarely there is hyperpyrexia. The question that confronts us here is, Can we produce the temperature in this disease without increasing cardiac weakness?

Generally speaking pneumonia is a self-limited disease, the crisis being reached usually before the end of the first week. Hence there is little need to attempt to lower temperature in a direct manner in the majority of instances. A temperature not exceeding $140^{\circ}$ F. calls for nothing more than frequent cold sponging of the surface and the exhibition of moderate doses of quinine, for example, 16 to 20 grains daily in divided doses. This drug has a slight antipyretic influence whilst it does not depress the heart, but rather supports it. Such internal antipyretics as phenacetine, antifebrin and antipyrin should be rigidly eschewed, since their good effects in combating high temperature is more than counterbalanced by the evil consequences in depressing the cardiac, respiratory, and vaso-motor nerve functions. Very high temperature demands cold to the surface either in the form of cold packs, or cold or gradually cooled baths. This method I have recently followed with very gratifying results. Dr. Bordman Reed, ${ }^{2}$ as the result of a statistical inquiry into various modes of treatment, concludes, "That water locally applied either by wet packs or in the form of baths, after the Brand method, is the most efficient single remedy or therapeutic measure for acute pneumonia."

We come now to the consideration of the second causal factor in the production of cardiac exhaustion, or that due to the barrier offered to the pulmonary circulation by the presence of the inflammatory exudate in the air vesicles.

In the first place it may be questioned whether the mere obliteration of a single lobe or even larger mass of ling tissue seriously interferes with the respiratory function, seeing that in animals as well as in man, life may be maintained for a long period after one entire lung has become impervious to atmospheric air. Neither do I believe that the power of the right ventricle would be inadequate if pneumonia consisted merely in a localized inflammatory process. This organ, therefore, is always predisposed to further anatomical changes, on account of the weakening influence of the ptomaines in the blood. It is readily seen then why, in many cases, the backward effect of the obstructive influences in the lungs are evidenced by the development of an overfilled and dilated condition of the right ventricle. The physical signs present, together with the signs of venous stasis, easily distinguish this condition from failure of the whole organ in consequence of intense systemic infection. The pulmonary second sound, which is at first accentuated, due to the strong action of the right ventricle and the strong recoil of blood in the pulmonary vessels, becomes progressively weaker until exceedingly feeble, pari passu with the diminution in the power of the right ventricle. This condition of the right heart is associated with an odematous condition of the lung, and great difficulty with the respiration, as a rule. The complexus of morbid conditions present in these cases is to be relieved by increasing right ventricular power on the one hand, and by removing the redundant liquid from the lesser circulation on the other hand. The latter indication is to be met by the repeated use of wet or dry cups. If in

2 Therapeutic Gazette, March and April. 1892. spite of these measures there is a definite increase in the severity of the symptoms, then free bleedings constitute sound practice. Venesection here acts in accordance with ordinary hydraulic principles, and only in this way. To attain to the same end some authors recommend dilating the arterioles by the use of the nitrites, etc., thus inviting the blood from the venous system to the arterial. If it were not for the fact, that in these cases too little blood reaches the left ventricle, the mode of treatment before alluded to should be sanctioned. In my opinion the physician's chief aim should be to propel more blood from the right to the left ventricle, after having removed as much of the obstacle in the lungs as possible, though to accomplish both objects i.e., dilating the arterioles and facilitating the cardio-pulmonary circulation without debilitating the heart would be the ideal treatment. I have recently attempted this, have administered digitalis with a view of increasing the capacity of the right ventricle for work and simultaneously have given small doses of nitro-glycerine for its effect in relaxing the blood vessel walls, with apparently good results. I was lead to use nitroglycerine in consequence of having learned the fact, that, in small doses, it is a cardiac stimulant rather than depressant, and hence, is not open to the same objections which I have urged against arterial relaxants as a class. Strychnia in large doses, and alcohol in moderate doses, must be added to the treatment. Doubtless strychnine is the most potent single agent to support the heart under these conditions. It is most efficient when administered hypodermically in doses of $\frac{1}{40}$ grain every four hours. Inhalations of oxygen are useful in these cases; they relieve dyspnoea and the frequency of the respirations by furnishing sufficient oxygen, in greatly diminished bulk, to ærate the blood. Thus they also accelerate the blood current through the lungs, since it is well known that respiratory impurities in the blood retard the flow of this circulating medium. Oxygen should be employed in this manner when the cyanosis and dyspnœa continue to grow definitely worse despite the use of alcohol, digitalis and strychnine. I have seen truly surprising results from its employment in well selected cases of acute lobar pneumonia in hospital practice.

The formation of cardiac thrombi in the course of the disease constitutes a more influential factor in the production of cardiac exhaustion than is generally supposed by writers on this subject. Doubtless they are often preceded by commencing heart failure, and often, too, are associated with odema of the lungs.

In acute lobar pneumonia the fibrin factors of the blood are greatly increased; a condition favoring their generation. And, to facilitate the same result, we have the over-filled state of the right heart as well as the sluggishness of the circulation in both heart and lungs. During a recent discussion of the subject of the treatment of lobar pneumonia before the Philadelphia County Medical Society, I took the position that "heart clot.s" are frequently followed by dilatation of the right ventricle, cyanosis and finally death. Two of the speakers, Drs. S. Solis-Cohen and Judson Daland, subsequently sustained me in this view. Dr. Daland stated that in the course of some examinations of the blood from patients ill of various diseases, he had occasion to examine blood taken from several cases of acute lobar pneumonia; and that when he attempted to examine specimens of this fluid 
it would coagulate in the capillary pipette with great rapidity, hence requiring special manipulations to make the investigations.

The symptoms vary with the rapidity of their production. When this is gradual, the signs and symptoms are not immediately urgent; the dyspnoea is increased, the respirations more hurried, the pulse becomes more frequent, finally irregular and intermittent. The physical signs are somewhat peculiar. The cardiac impulse is often irregular, while the area of the heart's percussion dulness extends to a point beyond the right edge of the sternum. On auscultation a systolic murmur is often heard over the xyphoid cartilage, which murmur is transmitted upward and toward the left. The physical signs referable to the right heart may arise independently of œdema of the lungs-a fact which goes to show that heart clots may be, most probably, the starting point of a series of conditions leading to a rapidly fatal issue. Dr. B. Ward Richardson long since directed attention to the value of the ammonium salts in this affection with a view to maintaining the fluidity of the blood; and in this opinion I heartily concur. If there be reason to suspect the presence of thrombi, the administration of the preparations of ammonia, though they offer little promise of giving favorable results, should be vigorously pushed in the hope that the clots may be dissolved.

Alcohol and digitalis are to be employed cautiously, while strychnine is to be administered hypodermatically in full physiological doses; and when collateral fluxion in the lungs with serious respiratory disturbance develop, oxygen inhalations, for the reasons previously adduced, must be employed.

In conclusion, I desire to re-affirm a few of the more salient points already rehearsed in your hearing. To the fact that there are three leading causes of heart insufficiency in acute lobar pneumonia, especial emphasis should, I think, be given. Among these, the baneful effect upon the heart of the toxic matter in the blood is the most pernicious, is a factor in every case, and is not unfrequently the sole cause of death. Cardiac exhaustion due to the action of these ptomaines can be differentiated and presents peculiar indications for treatment, specially useful being antiseptics, alcohol and strychnine.

In a large proportion of cases the right heart, already weakened on account of general infection, becomes further weakened and finally exhausted as the definite result of the obstruction opposed to the circulation in the lungs. In these instances the right ventricle is over-distended and congestive odema of more or less of the non-consolidated portion of the lung exists. This combination of conditions calls for relief in two directions. First, the removal of the abnormal blood pressure in the pulmonary ves: sels must be relieved either by cupping or free bleedings; the cardio-pulmonary circulation must be facilitated by using inhalations of oxygen, and strengthening the right ventricle, by administering digitalis generously, strychnine and alcohol. The use internally of the nitrites in small doses is to be advised, while the use of all other arterial relaxants is to be condemned, since the agents that accomplish this end tend to depress the heart.

The third, though perhaps, not the most unimportant cause of right ventricular exhaustion, are the cardiac thrombi, which are generated largely in consequence of the sluggish pulmonary circulation and the increased tendency to coagulation of the blood. Their presence is frequently demonstrable during the progress of the disease. For the puxpose of preventing their formation preparations of ammonium should be employed, since, as before indicated, they tend to preserve the fluidity of the blood. When "heart clots" of appreciable size exist, and even when their presence is only highly probable, this agent should be exhibited in liberal amount, though as before intimated it is quite doubtful whether the remedy is powerful to liquify these offending masses. Digitalis is to be used cautiously, alcohol in moderate doses and strychnia liberally.

Dr. Bailey, of Louisville, in opening the discussion remarked that he believed the treatment of croupous pneumonia depended almost if not entirely upon the management of the heart. He thought the essayist had given us a most admirable explanation of the heart's action and the course for the proper treatment of the disease. The proper means of reducing the temperature is of the greatest importance. Is there a remedy by which we can reduce it with as little reduction of the heart action as we can by the use of cold water? This preparation he thought we had in phenacetine. He was as willing, he said, to use it in this condition as he was cold water. He believed also that in that condition in which the avenues of the circulation are congested and it is recommended to abstract blood, good results have not been obtained from venesection since fifty or sixty years ago. He commended the ammonia preparations for the conditions indicated but thought they had no power to dissolve clots aIready formed, although they may prevent the formation of them. He recommended also the nitrite of amyl for opening up the capillaries and getting rid of obstructions, but the principal remedy is digitalis, which he thought was worth all other remedies combined for the relief of obstruction in the lungs.

Dr. Truax, of New York, desired to endorse Dr. Ander's paper, particularly what he had said on the subject of venesection. For a number of years, he said, I have been in the habit of extracting blood in conditions of great embarrassment to the circulation. I have done it experimentally. I have had the internes of the hospital pick out six or seven, or eight of the cases whom they thought most likely to die of heart failure and those who seemed to be the worst, and we have tried different forms of treatment over and over and over again. There is no doubt in my mind that there is any other means by which embarrassment of the circulation in the lungs can be removed and the heart enabled to do its work as by venesection. Stimulants will tide over the heart's action until the congestion is removed. I am sure that hundreds die every year who would be saved if they were bled.

Dr. Herrick, of Cleveland, stated that his line of inquiry would not agree with that of the essayist. He thought we were given too much to theory as to the causation of pneumonia. In this respect he confessed that he was somewhat adverse to the profession as to the theories of the causes of disease. He opposed the bacteriological origin of disease.

Dr. Greenlee, of Kentucky, expressed hearty endorsement and approval of the views of the last speaker. He saw no reason for the supposition that there is a micrococcus!

Dr. Pennell, of Ohio, thought that in some cases you must whip the tired heart up by the use of stimulants, whereas in other cases, venesection must be resorted to.

Dr. Didama, of New York, remarked that Neimeyer and other standard authors state that in some cases the congestion must be reduced by the removal of blood.

Dr. Musser, of Philadelphia, said that this is a subject which demanded from us a clear and full consideration. We ought to have clear ideas on the subject of pneumonia; and the clearer cut our ideas are, the better will we treat the disease. But if we are asked how to treat pneumonia, we can not lay down any rule. Our lines of treatment must be based upon the study of the individual case; nevertheless, we can form certain general ideas which we can keep in view. $\mathrm{He}$ was thoroughly convinced, and believed that he was in accord with modern pathology, that pneumonia is a specific infectious disease. Such being the case, we turn naturally to the form of treatment which might be considered specific; but such we do not as yet know. We have no specific means of combating this disease any more than we have for the other specific diseases, unless we except malaria. The treat- 
ment therefore resolves itself into the treatment of the inflammatory process, and there is no doubt about it that we have only three measures, actual, positive measures that we can always rely upon, for the combating of inflammation. They are cold, bloodletting and rest. Of the importance of cold locally, I am thoroughly impressed. I am also thoroughly in accord with the remarks that have been made on the use of bloodletting. I believe that in almost all cases it would be of advantage. It certainly would not do any harm and it might do a great deal of good. Advantages would arise in almost every case if bloodletting were practiced early. We must, however, be careful as to the indications for bloodletting. We must consider the physical signs, and not only these, but the condition of the pulse, the respiration and the temperature.

Absolute rest is important-not only the rest of the patient in bed, but the freedom from anxiety-perfect nursing is indicated. In securing this complete rest, opiates are demanded. I am always glad to give opium or some of its preparations. Possibly opium may help to allay inflammation as some pharmacologists think.

One. word, the speaker continued, with regard to the use of proper remedies. I think that strychnia is a proper remedy, and it may tide our patient over sometimes when they are sailing pretty close to the shore. I am in favor of nitro-glycerine, particularly if there is any tendency to heart failure or chronic parenchymatous nephritis. Of digitalis, I cannot say much. I would prefer alcoholics and preparations of ammonia. Of alcoholic stimulants, we must say that a certain time comes when champagne can be used instead of whisky, and I am sure that the use of this readily diffusible stimulant bas stood me in good stead.

We cannot estimate too highly the beneficial effect of good nursing. As to blood letting in the later stages, I am sorry to say that my experience has not been very good. In nearly all of my cases death has ensued in spite of the venesection. Oxygen, I am glad to endorse.

Dr. Anders, in closing the discussion supported the view that pneumonia is an acute infectious disease. In regard to the treatment, he said that so long as we have no specific antidote, we must simply endeavor to support the patient until the disease has subsided.

Afternoon Session.

The only papers read at the afternoon session were those on

\section{THE ANALOGY BETWEEN ACUTE IDIO- PATHIC PLEURITIS AND ACUTE ARTICULAR RHEUMATISM.}

$$
\text { BY E. L. SHURLY, M.D., }
$$$$
\text { OF DETROIT, MICH. }
$$

In the year 1887 , I read a paper bearing the above title before the Michigan State Medical Society, which at the time attracted very little attention. However, subsequent observation of these diseases has led me to think that too little importance has been placed upon this question; and for this reason, I venture now to bring the matter again to public notice.

Attention was first called to this relationship by Vallieux in 1854, and afterwards by Davis, of London, England; but without awakening much thought at that time or subsequently.

In the paper alluded to, the theoretical basis assumed of the identity of the two diseases was mainly twofold, on the one hand histological, because the pleura as well as the synovial and other serous membranes spring from the same primordial layer, the meso-blast or middle layer, and ultimately develop into the endothelial layer which is the lining of all closed cavities, and, on the other, because a correspondence between the phenomena characterizing the general history of the two diseases seems to exist.

This will become quite convincing if one takes the pains to carefully generalize and compare the various steps in the progress of the two diseases, idiopathic acute pleuritis and acute rheumatic arthritis. Besides, noting how frequently the pleural as well as the pericardial structures participate in the rheumatic affection, I am aware that such a complication of articular rheumatism is not recorded by medical writers as of common occurrence. Yet, I believe, with all due deference, that it is because it is so frequently overlooked, just as syphilitic pleuritis has been, until recently, so generally overlooked; nor would such a mishap appear unusual when we consider how insidjous and often localized may be an attack of fibrinous pleuritis. I certainly believe that a retrospective view of the clinical histories of cases of articular rheumatism by any practitioner present would recall certain signs of pleural implication, which, at the time, went unobserved, or were attributed to either secondary or concomitant manifestations, or cardiac complication.

I may mention such complaints as fugitive pain in the side, transient respiratory embarrassment, sudden exacerbations of temperature unattended by increased joint disturbance, etc.

Often, these phenomena are taken as denotive of peri-cardiac or cardiac implication, although close physical exploration fails to recognize the pathognomonic signs. Yet the mind is so intense upon the usual course of events, that failure to recognize the adventitious cardiac sounds, is attributed to lack of auditory power-so strong, in the best of human minds, is preconception.

It occurred to me a few years ago, after listening in vain for signs of cardiac disease in a given case, and when about to give up the search-but not the conviction-that I chanced to place my ear in the infra-axillary region and there detected a slight pleural friction sound, which, of course, determined the diagnosis, and which, I must say was amply corroborated by the subsequent course of the disease.

Besides these considerations we may apply as an additional proof the crucial test of treatment, a practice we are all familiar with, for the purpose of clearing away obscurity surrounding many cases of undetermined syphilitic disease. In this way one may often observe with great gratification the prompt check to the course of acute fibrinous and fibrinoserous pleuritis, and the rapidity with which the effused material is absorbed.

In illustrating the point, I would cite a very serious case which came under my observation lately in association with my colleague, Dr. Chapaton, one of our keenest observers, in which nearly the whole left pleural surface together with the pericardium was involved.

Without wearying you with details, suffice it to say, that under a so-called anti-rheumatic treatment principally-such as the salicylates-the disease rapidly declined in a manner, to say the least, not possible under the established plans of treatment adopted for this disease. In this case there were no arthritic manifestations, until about the tenth day, when the patient complained of pain and slight swelling of the right hip joint and vertebral joints.

In other cases which I have observed, one of the knee-joints seemed to be the elected spot. Although some cases where the pleura was the primary seat of disturbance, never showed any arthitic disease.

Koster, in a late article published in the Therapeutische Monatschr. says that the use of salicylates in the treatment of pleuritic effusions has not become as general as it should. He has treated thus thirtytwo cases, twenty-seven of which were primary and 